\title{
Vortex unbinding transition in nonequilibrium photon condensates
}

\author{
Vladimir N. Gladilin and Michiel Wouters \\ TQC, Universiteit Antwerpen, Universiteitsplein 1, B-2610 Antwerpen, Belgium
}

(Dated: April 28, 2021)

\begin{abstract}
We present a theoretical study of a Berezinskii-Kosterlitz-Thouless like phase transition in lattices of nonequilibrium photon condensates. Starting from linearized fluctuation theory and the properties of vortices, we propose an analytical formula for the critical point containing four fitting parameters, that captures well all our numerical simulations. We find that the ordered phase becomes more stable when driving and dissipation is increased.
\end{abstract}

Introduction - Thermalization of cavity photons by repeated absorption and emission by dye molecules [1] has led to photonic Bose-Einstein condensates [2 4] and presents an invitation to study photonic systems from a quantum fluid perspective [5]. The dimensional reduction implied by the microcavity structure, required to give the photon a nonzero rest mass, immediately raises the issue of the Hohenberg-Mermin-Wagner theorem that forbids BEC in two dimensions at finite temperature [6]. Experimentally, this has been avoided by a harmonic trapping potential, that modifies the density of states, and condensation in the ground state has been observed 2-4]. In this geometry, the condensate is however not spatially extended.

In extended two-dimensional interacting bose gases, there is a Berezinskii-Kosterlitz-Thouless (BKT) transition [6 9] that separates a phase with free vortices from a phase where all vortex-antivortex pairs are bound. For this phase transition to occur, interactions are crucial: in their absence, the vortex core size tends to infinity and vortices cease to be well defined excitations. At first sight, this seems to be fatal for the possibility of a phase transition in extended photon condensates that show negligible interactions except for a very slow thermal nonlinearity [10].

For photon condensates, the main deviation from the ideal bose gas comes from driving and dissipation. The confining mirrors are never perfect so that photon losses have to be compensated by continuous pumping of the dye molecules. We have shown recently that the nonlinear dynamics of driving and dissipation renders the vortex core size in a lattice of coupled photon condenstates finite [11]. Motivated by experiments on excitonpolaritons, it has already been numerically demonstrated that nonequilibrium interacting bose gases feature a BKT-like transition [12 14]. At the same time it has been shown that the phase dynamics is actually in the Kardar-Parisi-Zhang (KPZ) universality class, which has been argued to destroy the superfluid-like phase [15 17]. In practice, however, the KPZ physics can be limited to very large system sizes, so that in experimental systems the BKT-like physics dominates [12 14, 18].

We will show in this Letter that numerical classical field simulations on a finite lattice of photon condensates predict a BKT-like transition between states with and without free vortex-antivortex pairs. In addition, we will construct an analytical expression containing a few fitting constants that explains the numerically obtained dependence of the critical point on the system parameters.

Model - The simplest theoretical model to describe a lattice of coupled photonic cavities in the quantum degenerate regime is the generalized Gross-Pitaevskii equation (gGPE) [19]:

$$
\begin{aligned}
i \hbar \frac{\partial \psi(\mathbf{x}, t)}{\partial t} & =\frac{i}{2}\left[B_{21} M_{2}(\mathbf{x}, t)-B_{12} M_{1}(\mathbf{x}, t)-\gamma\right] \psi(\mathbf{x}, t) \\
- & (1-i \kappa) J \sum_{\mathbf{x}^{\prime} \in \mathcal{N}_{\mathbf{x}}} \psi\left(\mathbf{x}^{\prime}, t\right)+\sqrt{2 \mathcal{D}(\mathbf{x}, t)} \xi(\mathbf{x}, t) .
\end{aligned}
$$

Here $\gamma$ is the photon loss rate and $J$ the coupling between the nearest-neighbor cavities [20, 21]. The photons thermalize due to repeated absorption and emission by the dye with respective rate coefficients $B_{12}$ and $B_{21}$. The ground (excited) molecular state occupation is denoted by $M_{1(2)}$ satisfying at all times $M_{1}(\mathbf{x})+M_{2}(\mathbf{x})=M$, where $M$ is the number of dye molecules at each lattice site. The Kennard-Stepanov relation [22 24] gives rise to energy relaxation with dimensionless strength $\kappa=B_{12} \bar{M}_{1} /(2 T)$ (we set the Boltzmann constant $k_{B}=1$ ) [19]. The last term describes the spontaneous emission noise [25, 26] : $\mathcal{D}(\mathbf{x}, t)=B_{21} M_{2}(\mathbf{x}, t)$ and $\xi(\mathbf{x}, t)$ is Gaussian white noise with correlation function $\left\langle\xi(\mathbf{x}, t) \xi\left(\mathbf{x}^{\prime}, t^{\prime}\right)\right\rangle=\delta_{\mathbf{x}, \mathbf{x}^{\prime}} \delta\left(t-t^{\prime}\right)$. The evolution of the number of excited molecules due to interactions with the photons is opposite to the change in number of photons due to emission (both deterministic and stochastic), absorption and energy relaxation. In order to compensate for the loss of energy in the system, external excitation with a pumping laser is needed. Under the condition $J \ll T$, which assures that the occupations of all momentum states are much larger than one, the generelized Gross Pitaevskii classical field model (1) is valid for all the modes and there is no need to use a more refined quantum optical approach [27 29].

The noise in Eq. (1) provides a description of the density and phase fluctuations. For the simplest case of a single cavity, a crossover in the density fluctuations between a 'grandcanonical' regime with large fluctuations $\left(\delta n^{2} \sim\right.$ 
$\bar{n}^{2}$ ), for $\bar{n}^{2} \ll M_{\text {eff }}$, and a 'canonical' regime with small fluctuations $\left(\delta n^{2} \ll \bar{n}^{2}\right)$, for $\bar{n}^{2} \ll M_{\text {eff }}$, have been observed 30, 31]. Here, the 'effective' number of molecules is given by $M_{\mathrm{eff}}=\left(M+\gamma e^{-\Delta / T} / B_{21}\right) /[2+2 \cosh (\Delta / T)]$, where $\Delta$ is the detuning between the cavity and the dye zero-phonon transition frequency. For $e^{\Delta / T} \ll 1$, one has $M_{\mathrm{eff}} \approx \bar{M}_{2} \approx \eta M e^{\Delta / T}$ with $\eta=1+\gamma /(2 \kappa T)$.

Bogoliubov analysis - While the linear Bogoliubov theory [6, 19] breaks down in the vicinity of the BKT transition, that involves large phase differences between neighbouring cavities, it nevertheless forms a good starting point to obtain insight in the analytical dependence of the transition temperature on the system parameters. From the linearized dynamics of the density and phase fluctuations, one obtains the following equation that relates the phase fluctuations to the density-phase correlations [19]

$$
\kappa\left\langle\left|\delta \theta_{\mathbf{k}}\right|^{2}\right\rangle+\frac{1}{2 \bar{n}}\left\langle\delta \theta_{-\mathbf{k}} \delta n_{\mathbf{k}}\right\rangle=\frac{\mathcal{D}_{\theta}}{\epsilon_{\mathbf{k}}},
$$

where the phase noise is

$$
\mathcal{D}_{\theta}=\frac{B_{21} \bar{M}_{2}}{4 \bar{n}}=\frac{\eta \kappa T}{2 \bar{n}}
$$

and the dispersion is of the tight binding form: $\epsilon_{\mathbf{k}}=$ $2 J\left[2-\cos \left(k_{x}\right)-\cos \left(k_{y}\right)\right]$.

In equilibrium, invariance under time reversal $(\theta \rightarrow-\theta$ and $\delta n \rightarrow \delta n)$ ensures that the second term in Eq. (2) vanishes. In nonequilibrium photon condensates, time reversal symmetry breaks down and the density-phase correlator will play an important role in our discussion of the phase fluctuations that lead to the BKT transition.

At large momenta, the kinetic energy is much larger than the time reversal breaking rates that involve the pumping and losses. The density-phase correlations therefore become negligible at large $k$, so that the phase fluctuations assume their thermal equilibrium value. At low momenta on the other hand, the nonlinear dissipative dynamics kicks in and deviations from the thermal behavior appear. The crossover between nonlinear and ideal photon behavior occurs around the momentum [19, 32]

$$
k_{c}=\left[\frac{\gamma \kappa}{4 J}\left(1+\frac{\bar{n}^{2}}{\bar{M}_{2}}\right) \frac{\bar{n}^{2}}{\bar{M}_{2}}\right]^{1 / 6}\left(\frac{\eta T}{J \bar{n}}\right)^{1 / 3} .
$$

At low temperatures, where phase fluctuations are moderate, Eq. (21) is accurate for all momenta, but close to the BKT temperature the linear approximation breaks down and the system properties are determined by the full nonlinear equations. Even then, at momenta $k>k_{c}$, the linear relation (2) is expected to hold approximately.

In order to proceed further, we integrate (2) over all momenta [33] to obtain for the local fluctuations:

$$
\kappa\left\langle\delta \theta^{2}\right\rangle+\frac{1}{2 \bar{n}}\langle\delta \theta \delta n\rangle=\frac{\mathcal{D}_{\theta}}{2 \pi J}\left[c_{1}+\ln \left(\pi / k_{c}\right)\right],
$$

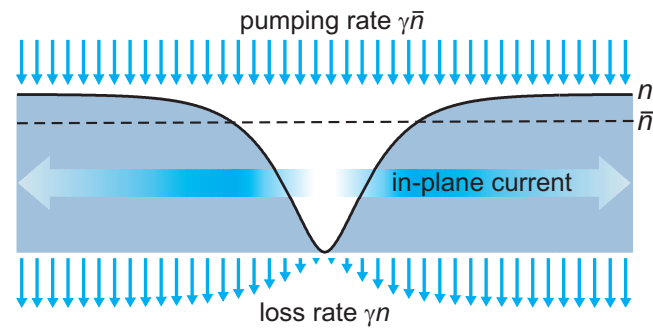

FIG. 1. Combination of a uniform pumping with losses proportional to the local density leads to outgoing particle flows from regions with reduced density.

where the constant $c_{1}$ approximates the contribution from the momenta $k<k_{c}$, where (2) breaks down. It is clear from the logarithmic dependence of (5) on $k_{c}$ that phase ordering is impossible in the absence of dissipative nonlinearity $\left(k_{c} \rightarrow 0\right)$, reflecting the well known fact that there is no phase transition for conservative noninteracting bosons in two dimensions.

Since phase fluctuations at the BKT transition are large, the parameter dependence of the transition point can be estimated with (5) by setting $\left\langle\delta \theta^{2}\right\rangle \sim 1$, provided that an estimate is available also for the the density-phase correlator. In order to obtain a first approximation, we restrict temporarily to one spatial dimension. Using partial integration to rewrite the density-phase correlator as $\langle\delta \theta \delta n\rangle=L^{-1} \int d x \delta \theta \delta n=-L^{-1} \int d x(\partial \theta / \partial x) \delta N$, where $\delta N=\int_{0}^{x} \delta n\left(x^{\prime}\right) d x^{\prime}$, it can be related to the current by use of the identity $\partial j_{x} / \partial x=-\gamma \delta n$. This continuity relation shows that regions with density suppression, such as a vortex core, behave as a source of currents (see Fig. 11) 11, 15, 34 36]. With $j_{x}=2 J \bar{n}(\partial \theta / \partial x)$, this yields

$$
\langle\delta \theta \delta n\rangle=\frac{1}{L} \frac{\gamma}{2 J \bar{n}} \int d x \delta N^{2}(x)=\frac{\gamma}{2 J \bar{n}}\left\langle\delta N^{2}\right\rangle .
$$

In order to estimate the expectation value $\left\langle\delta N^{2}\right\rangle$ close to the transition, one can first consider a plane density wave of amplitude $a \bar{n}$, for which $\left\langle\delta N^{2}\right\rangle \propto \bar{n}^{2} a^{2}$. At the transition, vortices have to nucleate, which requires in a continuum model density fluctuations with amplitude $\bar{n}$ and hence $a=1$. In a lattice geometry however, the vortex core can be 'in between' the lattice nodes and the density suppression smaller. In Ref. [11], the vortex core size was argued to roughly behave as $r_{0} \sim \sqrt{J / \gamma}$. Assuming an exponential wave function in the vicinity of the vortex center, with density profile $n(r)=\bar{n}\left[1-\exp \left(-r / r_{0}\right)\right]^{2}$, one can estimate the minimal density modulation depth sufficient to nucleate a vortex in the center of a plaquette as

$$
a=2 e^{-c_{3} \sqrt{\gamma / J}}-e^{-2 c_{3} \sqrt{\gamma / J}},
$$

where a constant $c_{3} \sim 1$ was introduced. From the above arguments, we come to the following estimate for the 
dependence of the density-phase correlator on the system parameters close to the transition point: $\langle\delta \theta \delta n\rangle=$ $2 c_{2} \bar{n} a^{2} \gamma / J$, with $c_{2}$ an additional fitting parameter.

Using $\left\langle\delta \theta^{2}\right\rangle=c_{4} \sim 1$ together with the above estimate of $\langle\delta \theta \delta n\rangle$ allows to rewrite Eq. (5) as a relation for the critical parameters:

$$
\frac{J \bar{n}}{T}=\frac{\eta \kappa}{4 \pi} \frac{c_{1}+\ln \left(\pi / k_{c}\right)}{c_{4} \kappa+c_{2} a^{2} \gamma / J}
$$

where $k_{c}$ and $a$ are given by Eqs. (4) and (7). Because of the quite handwaiving arugments that have led us to relation (8), it cannot be expected to hold exactly. Nevertheless, we will show below that it offers a good description of the critical point extracted from numerical simulations for the following values of the fitting parameters: $c_{1}=3.56, c_{2}=0.132, c_{3}=1.22, c_{4}=0.470$.

Figure 2 shows the variations of the dimensionless coupling constant $J \bar{n} /(\eta T)$ according to Eq. (8) as a function of the energy relaxation $\kappa$ and the ratio of losses to hopping $\gamma / J$ (note that both axes are logarithmic) for three values of the number of photons per cavity. We always restricted the dissipation strength to $\gamma / J<2$ in order to keep a resolved photon dispersion.

Even though these parameters vary by orders of magnitude, the variations in the coupling constant are quite moderate. For large photon numbers (canonical regime, lower panel), the coupling parameter is close to 0.6 except for small $\kappa$, where nonequilibrium effects are strongest. For smaller numbers of photons (grandcanonical regime), a larger coupling constant is needed in general and its variations are enhanced.

It is instructive to compare our relation (8) to the equilibrium BKT transition. The most elementary approximation is the reduction of the lattice Bose gas to the $\mathrm{XY}$ model, obtained by ignoring the density degree of freedom. The critical temperature has been determined to be $J \bar{n} / T=0.56$ [37], which is close to the value that we obtain in a large part of parameter space in the deep canonical regime, see Fig. 2(c).

In the limit of small $\gamma$, the second term in the denominator in Eq. (8), originating from the density-phase correlator, can be neglected and we obtain for the critical coupling parameter $J \bar{n} / T=\eta \ln \left(e^{c_{1}} \pi / k_{c}\right) /\left(4 \pi c_{4}\right)$, whose form is reminiscent of the transition point of the weakly interacting Bose gas [9]. The main differences with the equilibrium case are that the crossover momentum $k_{c}$ is now determined by losses instead of interactions and the appearance of the excess noise factor $\eta$. It is worth noting that in the limit of small $\gamma$, where $\eta \rightarrow 1$, the prefactor of the logarithm is in our fit equal to $1 /\left(4 \pi c_{4}\right) \approx 1 /(1.88 \pi)$, close to the equilibrium prefactor of $1 /(2 \pi)[9]$.

Formula (8) is also reminiscent of the heuristic expression $D_{\mathrm{BKT}} / n_{\mathrm{BKT}} \approx \kappa+0.003 c$, which has been shown to approximate fairly well the numerical results for the critical noise-to-density ratio as a function of $\kappa$



FIG. 2. Dimensionless coupling constant $J \bar{n}(\eta T)^{-1}$, given by Eq. (8), as a function of $\kappa$ and $\gamma / J$ for three different values of $\bar{n}^{2} / \bar{M}_{2}$.

and the 'nonequilibrium parameter' $c \propto \gamma$ in interactingpolariton condensates [38].

Numerical results - Numerical simulations of the full gGPE (1) for an array of $100 \times 100$ cavities with periodic boundary conditions were done as explained in Ref. [19] and the location of the critical point was determined as in Ref. 38]: after a long time evolution in the presence of noise, the system was evolved without noise for a short time $(\sim 10 \mathrm{~ns})$ before checking for the presence of vortices. This noiseless evolution gives the advantage of cleaning up the photon phase while it is too short for the unbound vortex-antivortex pairs to recombine. The propensity for their recombination is reduced [14] with respect to the equilibrium case thanks to outgoing radial currents that provide an effective repulsion between vortices and antivortices [15]. If no vortices are present in the final photon field, the system is said to be in the ordered phase; when vortex pairs are present, it is denoted as disordered.

Because the presence of vortices and antivortices is susceptible to statistical fluctuations, the numerical error on the transition point is not only due to our finite steps in parameter values, but also due to statistical uncertainty. The stochastic contribution to the error bar is hard to quantify precisely, but the analysis of many realizations of the dynamics allowed us to conclude that the statistical error bars are typically not larger than the symbol 


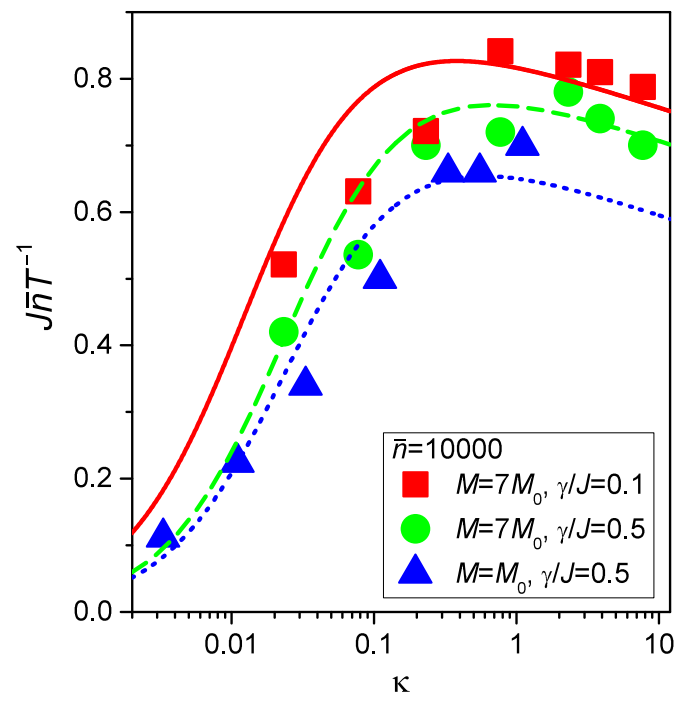

FIG. 3. Numerically determined dimensionless coupling strength $J \bar{n} T^{-1}$ at the BKT transition (symbols) as a function of $\kappa$ for different values of $M$ and $\gamma / J$. Here, $M_{0}=10^{9}$, $\Delta / T=-5.2$. The curves correspond to Eq. (8).

sizes in the figures.

As a first example of the parameter dependence of the critical coupling, we show in Fig. 3 $J \bar{n} / T$ as a function of $\kappa$, that was varied by changing both $M$ and $B_{21}$. The numerically obtained results are indicated with the symbols (symbols of the same type and color correspond to the same $M$ but different $B_{21}$ ) and the fits with relation (8) are shown with full lines. Good correspondence is observed over the whole range of $\kappa$, throughout which the critical coupling varies by one order of magnitude. The initial rise and subsequent saturation is clear from the explicit $\kappa$-dependence in Eq. (8). As can be seen from the denominator in Eq. (8), in the regime of small $\kappa$ the pumping and losses, proportional to $\gamma$, are dominant. The reduction of the critical coupling at small $\kappa$ can therefore be interpreted as an increased robustness of the ordered phase due to driving and dissipation, in analogy with Ref. 38]. The decrease of $J \bar{n} / T$ at large values of $\kappa$ originates from the $k_{c}$-dependence on $\kappa$, while the increase with $M$ is due to the dependence of $k_{c}$ on $\bar{M}_{2} \propto M$.

The dependence of the critical coupling on the number of photons per lattice site is illustrated in Fig. 4. Our relation (8) again captures most of the parameter dependence. According to Eq. (8), the decrease of the critical coupling parameter with the number of photons is due to the increase of the crossover momentum with increasing $\bar{n}$. This trend is reflected in the numerical results, but the numerical $\bar{n}$-dependence shows some features that are not entirely reproduced by our formula and require a more sophisticated theoretical approach. At first sight, it could be surprising that a phase transition still exists down to photon numbers as small as $\bar{n}=1000$,

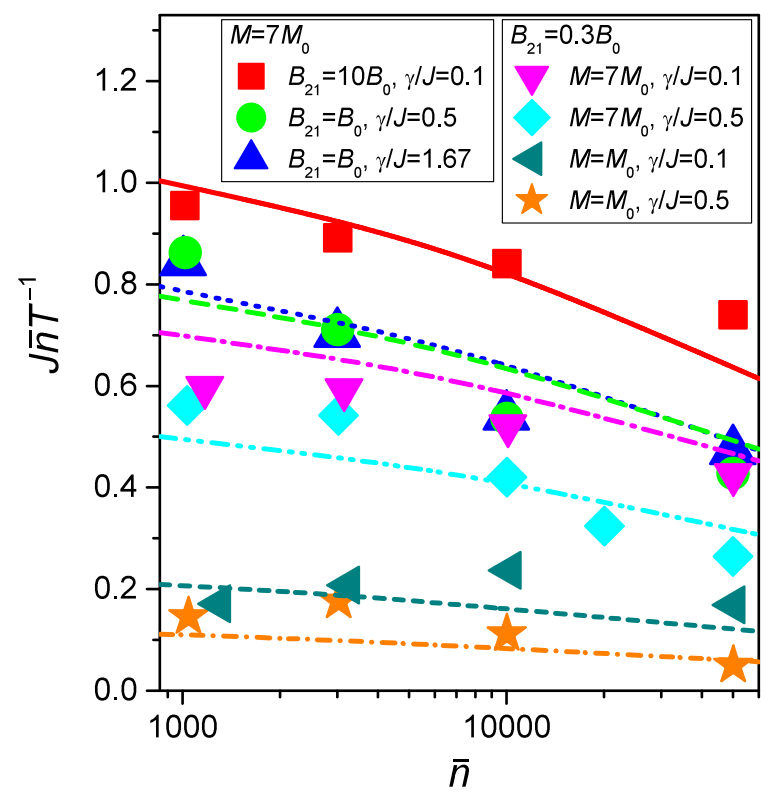

FIG. 4. Numerically determined dimensionless coupling strength $J \bar{n} T^{-1}$ at the BKT transition (symbols) as a function of $\bar{n}$ for different values of $M, B_{21}$ and $\gamma / J$. Here, $M_{0}=10^{9}, B_{0}=10^{-7} \mathrm{meV}, \Delta / T=-5.2$. The curves correspond to Eq. (8).

well in the grandcanonical regime with $\bar{n}^{2} / M_{\text {eff }}=0.18$ and 0.026 for $M=10^{9}$ and $M=7 \times 10^{9}$, respectively. Our explanation is that for sufficiently strong coupling between the cavities, a subset of $N$ cavities behaves collectively, thereby increasing the effective $n^{2} / M_{\text {eff }}$ linearly with $N$.

At $\gamma / J<1$ the critical coupling decreases with increasing $\gamma / J$ (compare magenta to cyan or dark-cyan to orange symbols/lines in Fig. 4, see also Fig. 2). For $\gamma / J \ll \kappa$ this decrease is determined by the behavior of $\ln \left(\pi / k_{c}\right)$ in Eq. (8), while for larger $\gamma / J$ the effect of the term $c_{2} a^{2} \gamma / J$ dominates. In the case of $\gamma / J \sim 1$, where the vortex core size is comparable to the intercavity spacing and the density modulation depth $a$ required for the BKT transition is significantly reduced, the aforementioned decrease of the critical coupling with increasing $\gamma / J$ can be fully canceled or even reversed due to a strong decrease of $a^{2}$ in the term $c_{2} a^{2} \gamma / J$ (compare green to blue symbols/lines in Fig. 4, see also Fig. 21).

Conclusions and outlook - Our numerical and analytical investigations of a lattice nonequilibrium BoseEinstein condensate of noninteracting photons have shown that a BKT-like transition exists between states with and without unbound vortex-antivortex pairs. According to Eq. (8), the vortex-free phase is actually stabilized by driving and dissipation. Our findings are in line with previous numerical studies [12, 13, 38] of nonequilibrium polariton condensates, where interactions were included. 
The experimental verification of our prediction for the spontaneous creation of vortices and antivortices should be possible by directly measuring the phase profile of a photon condensate by interferrometry as used for the observation of phase jumps of localized photon condensates [39].

In our numerics, we have not found evidence for the destruction of the ordered phase as was predicted on the basis of the description of the phase dynamics by the nonlinear KPZ model [15-17]. This could be due to our finite simulation area, but the interplay of BKT and KPZ physics in nonequilibrium condensates $40-42$ should be explored further. The stability of the vortex free phase could be important for potential applications to analog optical computations [43 48] with photon condensates [21]. From the side of fundamental physics, it will also be interesting to study the dynamics of a photon condensate lattice after a density quench through the phase transition [18, 49].

Acknowledgements - We are grateful to Jan Klaers, Fahri Öztürk, Julian Schmitt, Martin Weitz, Iacopo Carusotto, Jacqueline Bloch and Wouter Verstraelen for stimulating discussions. VG was financially supported by the the FWO-Vlaanderen through grant nr. G061820N.

[1] J. Klaers, F. Vewinger and Martin Weitz, Nat. Phys. 6, $512(2010)$.

[2] J. Klaers, J. Schmitt, F. Vewinger, and M. Weitz, Nature 468, 545 (2010).

[3] J. Marelic, L. F. Zajiczek, H. J. Hesten, K. H. Leung,E. Y. X. Ong, F. Mintert and R. A. Nyman, N. J. Phys. 18, 103012 (2016).

[4] S. Greveling, K. L. Perrier and D. van Oosten, Phys.Rev. A 98, 013810 (2018).

[5] I. Carusotto and C. Ciuti, Rev. Mod. Phys. 85, 299 (2013).

[6] L. P. Pitaevski and S. Stringari, Bose-Einstein condensation (Oxford University Press, 2016).

[7] V. L. Berezinskii, Sov. Phys. JETP 32, 493 (1971).

[8] J. M. Kosterlitz and D. J. Thouless, J. Phys. C: Solid State Phys. 6, 1181 (1973).

[9] N. Prokof'ev, O. Ruebenacker, and B. Svistunov Phys. Rev. Lett. 87, 270402 (2001).

[10] H. Alaeian, M. Schedensack, C. Bartels, D. Peterseim and M. Weitz, N. J. Phys. 19, 115009 (2017).

[11] V. N. Gladilin and M. Wouters, Phys. Rev. Lett. 125, 215301 (2020).

[12] G. Dagvadorj, J. M. Fellows, S. Matyjaskiewicz, F. Marchetti, I. Carusotto and M. H. Szymanska, Phys. Rev. X 5, 041028, (2015).

[13] D. Caputo, D. Ballarini, G. Dagvadorj, C. S. Muñoz, M. De Giorgi,L. Dominici, K. West, L. N. Pfeiffer, G. Gigli, F. P. Laussy, M. H. Szymańska and D. Sanvitto, Nat. Mat. 17,145 (2018).

[14] V. N. Gladilin and M. Wouters, J. Phys. A 52, 1751 (2019).

[15] G. Wachtel, L. M. Sieberer, S. Diehl and E. Altman,
Phys. Rev. B 94, 104520 (2016).

[16] L. M. Sieberer, G. Wachtel, E. Altman and S. Diehl, Phys. Rev. B 94, 104521 (2016).

[17] E. Altman, L. M. Sieberer, L. Chen, S. Diehl, and J. Toner, Phys. Rev. X 5, 011017 (2015).

[18] P. Comaron, G. Dagvadorj, A. Zamora, I. Carusotto, N. P. Proukakis, and M. H. Szymańska, Phys. Rev. Lett. 121, 095302 (2018).

[19] V. N. Gladilin and M. Wouters, Phys. Rev. A 101, 043814 (2020).

[20] D. Dung, C. Kurtscheid, T. Damm, J. Schmitt, F. Vewinger, M. Weitz and J. Klaers, Nat. Phot. 11, 565 (2017).

[21] B. Kassenberg, M. Vretenar, S. Bissesar, J. Klaers, arXiv:2001.09828

[22] E. H. Kennard, Phys. Rev. 11, 29 (1918).

[23] B. I. Stepanov, Dokl. Akad. Nauk SSSR 112, 839 (1957). [Sov. Phys. Dokl. 2, 81 (1957)].

[24] P. Moroshkin, L. Weller, A. Saß, J. Klaers and M. Weitz, Phys. Rev. Lett. 113, 063002 (2014).

[25] C. Henry, IEEE Journal of Quantum Electronics 18, 259(1982).

[26] W. Verstraelen and M. Wouters, Phys. Rev. A 100, 013804 (2019).

[27] P. Kirton and J. Keeling, Phys. Rev. Lett. 111, 100404(2013).

[28] P. Kirton and J. Keeling, Phys. Rev. A 91, 033826 (2015).

[29] P. Kirton and J. Keeling, Phys. Rev. A 93, 013829 (2016).

[30] J. Klaers, J. Schmitt, T. Damm, F. Vewinger,and M. Weitz, Phys. Rev. Lett. 108, 160403 (2012).

[31] J. Schmitt, T. Damm, D. Dung, F. Vewinger, J. Klaers and M. Weitz, Phys. Rev. Lett. 112, 030401 (2014).

[32] The only difference with the expression in Ref. [19] is that there approximations $1 \ll \bar{n}^{2} / \bar{M}_{2}$ and $\eta \approx 1$ were made. These are relaxed here.

[33] We approximate the tight binding lattice dispersion by its low energy quadratic behavior in order to obtain a simpler analytical expression. We have checked that using the full dispersion has only minor effects on the subsequent analysis.

[34] V. N. Gladilin and M. Wouters, New J. Phys. 19, 105005 (2017).

[35] I. S. Aranson and L. Kramer, Rev. Mod. Phys. 74, 99 (2002).

[36] K. Staliunas, V. J. Sánchez-Morcillo, Transverse Patterns in Nonlinear Optical Resonators (Springer, Berlin, 2003).

[37] Yun-Da Hsieh, Ying-Jer Kao and A. W. Sandvik, J. Stat. Mech. P09001 (2013).

[38] V. N. Gladilin and M. Wouters, Phys. Rev. B 100, 214506 (2019).

[39] J. Schmitt, T. Damm, D. Dung, C. Wahl, F. Vewinger, J. Klaers, and M. Weitz Phys. Rev. Lett. 116, 033604 (2016).

[40] D. Squizzato, L. Canet and A. Minguzzi, Phys. Rev. B 97, 195453 (2018).

[41] V. N. Gladilin, K. Ji, and M. Wouters, Phys. Rev. A 90, 023615 (2014).

[42] L. He, L. M. Sieberer, E. Altman, and S. Diehl, Phys. Rev. B 92, 155307 (2015).

[43] N. G. Berloff, M. Silva, K. Kalinin, A. Askitopoulos, J. D. Töpfer, P. Cilibrizzi, W. Langbein and P. G. Lagoudakis, 
Nat. Mater. 16, 1120 (2017)

[44] P. Lagoudakis and N. G. Berloff, New J. Phys. 19, 125008 (2017).

[45] K. Kalinin, P. G. Lagoudakis, N. G. Berloff, Phys. Rev. B 97, 094512 (2018).

[46] P. L. McMahon, A. Marandi, Y. Haribara, R. H, C. Langrock, S. Tamate, T. Inagaki, H. Takesue, S. Utsunomiya, K. Aihara, R. L. Byer, M. M. Fejer and H. Mabuchi, Y.
Yamamoto, Science 354614 (2016).

[47] Y. Yamamoto, K. Aihara, T. Leleu, K. Kawarabayashi, S. Kako, M. Fejer, K. Inoue and H. Takesue, NPJ Quant. Inf. 3, 49 (2017).

[48] C. Tradonsky, I. Gershenzon, V. Pal, R. Chriki, A. A. Friesem, O. Raz and N. Davidson, Science Advances. 5, 4530 (2019).

[49] M. Kulczykowski and M. Matuszewski, Phys. Rev. B 95, 075306 (2017). 